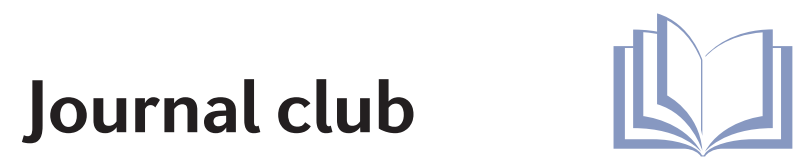

\section{TINKERING WITH NATURE}

As a postdoctoral researcher,

I attended a talk by Francis Crick, who described nature as "a tinkerer". In evolution, basic structures are often altered, through the addition of units and novel functions, to generate more complex systems. This process is reminiscent of my children using small Lego blocks to build complicated structures that expand in all dimensions over the course of the day. If we were to design a biological process or structure, we might devise a very different construction to that built by nature. As biochemists, we are fascinated by complex structures and processes, but our main goal is to understand how they function at the molecular level. To do this, we need to tinker with processes and structures, often 'reverse-engineering' them back to the basics.

An excellent example of biochemical reverse-engineering was published by Görlich and Rapoport in
1993. This work presented the purification of the Sec61 complex, a protein translocase, from solubilized membranes. The purified complex was reconstituted into liposomes and shown to transport precursor proteins across the membrane. Although today we know that the Sec61 complex is the main gate into the endoplasmic reticulum, in 1993 this was far from the common view. In addition, it was at this time unclear that a complex consisting of three subunits, together with the signal-recognition particle receptor, could actually transport a protein in a co-translational manner, a fact also demonstrated by Görlich and Rapoport. Subsequently, in 1997, Beckmann et al. presented the first glimpse of this process by using cryo-electron microscopy to reveal the structure of the ribosome-Sec61 complex from purified components.

Thus, the use of a reductionistic approach, in 1993, to rebuild a biological process from a minimal set of pure components, played an instrumental part in our understanding of protein transport across biological membranes. This work, and related studies on the bacterial counterpart, SecYEG, finally led to the resolution of the crystal structure of the SecYEG, by Van den Berg et al., and provided the detailed view of the Sec translocase that is available in many textbooks today.

Interestingly, despite this tremendous gain of knowledge regarding the composition and structure of the translocase over the previous decades, many mechanistic questions still remain, and ample tinkering endeavors await.

Peter Rehling Abteilung für Biochemie II, Universität Göttingen, D-37073 Göttingen, Germany; and Max Planck Institute for Biophysical Chemistry, 37077 Göttingen, Germany. e-mail: peter.rehling@medizin.uni-goettingen.de The author declares no competing financial interests.

ORIGINAL RESEARCH PAPERS Görlich D. \& Rapoport T. A. Protein translocation into proteoliposomes reconstituted from purified components of the endoplasmic reticulum membrane. Cell 75, 615-630 (1993) | Beckmann, R. et al. Alignment of conduits for the nascent polypeptide chain in the ribosome-Sec61 complex. Science 278, 2123-2126 (1997) | Van den Berg, B. et al. X-ray structure of a proteinconducting channel. Nature 427, 36-44 (2004) 